\title{
Sidetap \& Slingshot Gestures on Unmodified Smartwatches
}

\author{
Hui-Shyong Yeo ${ }^{1}$ \\ hsy@st-andrews.ac.uk \\ Juyoung Lee ${ }^{2}$ \\ ejuyoung@kaist.ac.kr \\ Andrea Bianchi ${ }^{3}$ \\ Aaron Quigley ${ }^{1}$ \\ andrea@kaist.ac.kr \\ aquigley@acm.org \\ ${ }^{1}$ School of Computer Science, University of St Andrews, Scotland, United Kingdom \\ ${ }^{2}$ Graduate School of Culture Technology, KAIST, Republic of Korea \\ ${ }^{3}$ Department of Industrial Design, KAIST, Republic of Korea
}

\begin{abstract}
We present a technique for detecting gestures on the edge of an unmodified smartwatch. We demonstrate two exemplary gestures, i) Sidetap - tapping on any side and ii) Slingshot pressing on the edge and then releasing quickly. Our technique is lightweight, as it relies on measuring the data from the internal Inertial measurement unit (IMU) only. With these two gestures, we expand the input expressiveness of a smartwatch, allowing users to use intuitive gestures with natural tactile feedback, e.g., for the rapid navigation of a long list of items with a tap, or act as shortcut commands to launch applications. It can also allow for eyes-free interaction or subtle interaction where visual attention is not available.
\end{abstract}

\section{Author Keywords}

Smartwatches; tapping gesture; sidetap; slingshot

\section{ACM Classification Keywords}

H.5.2. Information interfaces and presentation: User interfaces - Input devices and strategies

\section{INTRODUCTION}

Wrist-worn wearables such as smartwatches and fitness trackers are of increasing interest in both research and commercial deployment. However, such devices rely on small touch screens or a few physical buttons for interaction. This can be difficult for the user due to the limited input expressiveness and the requirement for direct sight and visual attention.

Here, we propose a new technique for detecting i) Sidetap and ii) Slingshot gestures, which are i) tapping on any side and ii) pressing on the edge and then releasing quickly. These two simple gestures allow us to expand the input expressiveness of wristworn devices beyond screen or button input. In addition, by focusing on "interaction on the edge", we allow for eyesfree interaction where visual attention is not available.

Our contribution is a simple, yet effective detection technique, which does not rely on classification methods that are computationally expensive such as machine learning or template matching. We employ only the built-in IMU data directly, thus making our technique suitable for deployment

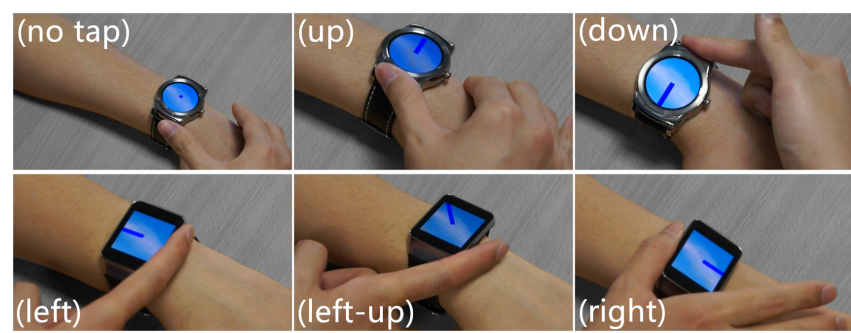

Figure 1. Side tapping on both circular and square watches. The blue bar shows the direction of force applied.

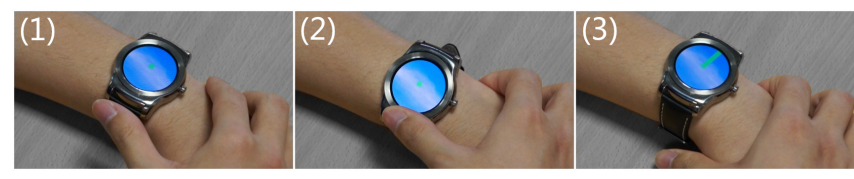

Figure 2. Slingshot in action (1) not touching (2) pressing down (3) quick release. The green bar shows the direction of shot.

on wearable devices with limited computing resources. Finally, we demonstrate the implementation of our prototype on a square and a circular Android smartwatch.

\section{RELATED WORK}

Previous research has attempted to extend smartwatch interaction to support in-air [6], around device [2, 4] and mechanical $[5,7]$ gesture, which each typically require hardware modification. Xiao et al. [7] and SkinWatch [5] enhance the interaction by supporting tilt, twist and pan on the watchface with joysticks or photo-reflective sensors. EdgeSense [4], SkinButtons [2] and zSense [6] expand the input area to the side of the watch, skin surface beside the watch and mid-air area around the watch, using capacitive or infrared sensors.

There are also approaches $[3,8]$ that are able to infer side or back tapping motion on the device without requiring external hardware. However, these have only been demonstrated on a smartphone, not on smartwatch, as these methods [3, 8] rely on machine learning to classify the tap, which might be difficult for wearables with limited computing resources.

\section{DESIGNING SIDETAP AND SLINGSHOT}

Our gesture design is motivated by five goals: i) No hardware or modification required. ii) Low processing requirement for running on smartwatch natively. iii) Accurate detection and robust to false triggering. iv) Intuitive and natural tactile feedback. v) Support for eyes-free interaction. The end result is Sidetap \& Slingshot which support tapping and quick release gestures from all 8 directions (4 corners and on 4 edges). 

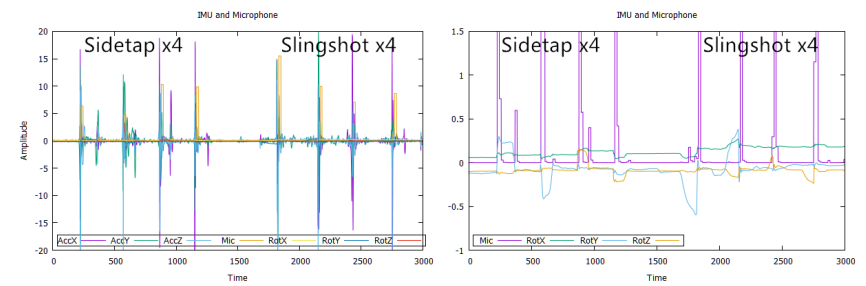

Figure 3. A Sidetap or Slingshot gesture causes spike in the acceleration and microphone input, which can be extracted via peak detector. Note the difference between the two gestures - the rotation value changes before impact (sidetap) or after impact (slingshot).

\section{Working Principle}

Wristworn devices are typically held in place by a strap to areas of human skin. Both the elasticity of human skin and any strap act to return the device to its original position (akin to RubberEdge [1]), when they encounter a light force.

As a result of these two key characteristics, when a watchface is hit or tapped from one direction, it will move in the opposite direction, tilting the watchface slightly. By measuring this subtle change in the orientation value, we can determine the opposite direction of the applied force. In addition, when a tap or quick release occurs, there exist a sharp peak in both the acceleration data and microphone input amplitude.

\section{Implementation}

We implemented a prototype on two unmodified Android smartwatches (Samsung Gear Live and LG Urbane), using Android Wear SDK, as shown in Figure 1 and 2. Linear acceleration and a rotation vector of the watch is polled at about $100 \mathrm{~Hz}$ while microphone input is sampled at $16 \mathrm{kHz}$.

The changes in rotation vector (Figure 3 right) before and after trigger are measured and converted to $\mathrm{x}-\mathrm{y}$ coordinate linearly. This is used to determine the direction and which gesture. For Sidetap, the rotation changes after impact while for Slingshot, the rotation changes before impact. A short delay is used because the IMU data is very noisy during impact.

In addition, we need a robust way to trigger the gesture detection. Unfortunately, the side/rim of current smartwatches is not touch sensitive. After some experiments, we chose to employ a sensor fusion approach of detecting a sharp peak in both the acceleration and microphone input, as shown in Figure 3 left. This has proved to be quite robust. In addition, to avoid falsely triggering, the following heuristics are used - i) orientation of the watch should not fluctuate beyond a small threshold during or before the trigger (due to unintentional arm movement). ii) when microphone input is too loud (due to background noises or speaking), fall back to using linear acceleration peak only.

\section{EXAMPLE APPLICATIONS}

In a file explorer (Figure 4) with a long list of items, a top/bottom tap can skip to the first/last item. Sidetap gesture also allows shortcut command, e.g., left tap to start a timer or right tap to start a music player. Slingshot is particularly suitable for gaming purposes such as the "Angry bird" game (Figure 4), because of the realistic and natural feedback.

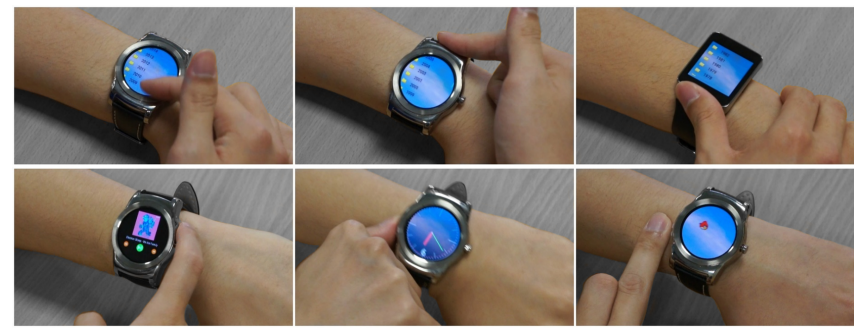

Figure 4. Example: (top) file explorer (bottom) shortcut and angry bird.

\section{CONCLUSION, LIMITATION AND FUTURE WORK}

We presented a new technique for detecting Sidetap \& Slingshot gestures on unmodified smartwatches. Our technique is lightweight yet effective, using only the built-in IMU data without heavy processing such as machine learning.

In current prototype, there are still some miss triggering. To reduce false triggering due to unintentional arm movement, we use a high threshold for the peak detection, thus requiring a strong tap from the user to register as input. This is a trade-off, as reducing threshold reduces miss triggering but increases false triggering, and vice-versa. Because there is only a single microphone embedded in the watch, the amplitude of microphone input is also different when tapping from different side (e.g., right side is higher on Gear Live).

In future work, we aim to improve the robustness and then perform a thorough user study to evaluate the system. Furthermore, instead of binary state detection (tap or not tap), we aim to detect the pressure level and the impact force.

\section{REFERENCES}

1. Casiez, G., Vogel, D., Pan, Q., and Chaillou, C. Rubberedge: Reducing clutching by combining position and rate control with elastic feedback. In UIST '07,

2. Laput, G., Xiao, R., Chen, X. A., Hudson, S. E., and Harrison, C. Skin buttons: Cheap, small, low-powered and clickable fixed-icon laser projectors. In UIST' 14 ,

3. McGrath, W., and Li, Y. Detecting tapping motion on the side of mobile devices by probabilistically combining hand postures. In UIST '14,

4. Oakley, I., and Lee, D. Interaction on the edge: Offset sensing for small devices. In $\mathrm{CHI}$ ' 14 ,

5. Ogata, M., and Imai, M. Skinwatch: Skin gesture interaction for smart watch. In $A H^{\prime}$ ' 15 ,

6. Withana, A., Peiris, R., Samarasekara, N., and Nanayakkara, S. zsense: Enabling shallow depth gesture recognition for greater input expressivity on smart wearables. In $\mathrm{CHI}$ '15,

7. Xiao, R., Laput, G., and Harrison, C. Expanding the input expressivity of smartwatches with mechanical pan, twist, tilt and click. In $\mathrm{CHI}$ '14,

8. Zhang, C., Guo, A., Zhang, D., Southern, C., Arriaga, R., and Abowd, G. Beyondtouch: Extending the input language with built-in sensors on commodity smartphones. In IUI'15, 